\title{
Spectrally-resolved dynamics of two-state lasing in quantum-dot lasers
}

\author{
M.-H. Mao ${ }^{\text {a }}$ L.-C. Su ${ }^{\text {a }}$ K.-C. Wang ${ }^{\text {a }}$, W.-S. Liu ${ }^{\text {b }, ~ P .-C . ~ C h i u ~}{ }^{\text {b }}$ J.-I. Chyi ${ }^{\text {b }}$ \\ ${ }^{a}$ Department of Electrical Engineering, Graduate Institute of Electronics Engineering, and \\ Graduate Institute of Electro-Optical Engineering, National Taiwan University \\ No.1, Sec. 4, Roosevelt Road, Taipei, Taiwan 106, R.O.C. \\ Phone: 886-2-23635251-350,FAX: 886-2-23677467,E-mail:f92941030@ntu.edu.tw \\ ${ }^{b}$ Department of Electrical Engineering, National Central University \\ No.300, Jhongda Rd., Jhongli City, Taoyuan, Taiwan, R.O.C.
}

\begin{abstract}
Spectrally-resolved dynamics of two-state lasing in quantum-dot lasers is experimentally demonstrated for the first time in this study. The onset of excited-state lasing prior to ground-state lasing is consistent with our theoretical prediction.
\end{abstract}

Quantum-dot (QD) lasers have attracted much attention in recent years due to their superior properties, such as ultra-low and temperature-stable threshold current density, high-speed operation, and low frequency chirping [1]. Recently, experimental observation of simultaneous lasing in steady state from ground states (GS) and excited states (ES) in QD lasers has been reported [2,3]. This effect was studied also theoretically, both in steady state and in transient behavior [4]. However, to our knowledge, there is no experimental investigation about the individual transient responses of GS and ES, respectively, from two-state-lasing QD lasers up to now. In this work, spectrally-resolved dynamics of two-state lasing in QD lasers is experimentally demonstrated for the first time. Theoretical simulation based on a rate-equation model [5] is also carried out in order to analyze the measured results in details.

The sample was grown by MBE with five layers of InAs QDs capped by $7 \mathrm{~nm}$-thick $\operatorname{In}_{0.17} \mathrm{Ga}_{0.83}$ As layer as active medium and GaAs as spacer layer. The ridge waveguide laser structure was processed into $5 \mu \mathrm{m}$ width and was cleaved at both facets to form a laser cavity of $2 \mathrm{~mm}$ length. The device was then driven by an electrical pulse source with $150 \mathrm{ps}$ rise time and pulse duration of several ns for both static and dynamic measurements. For spectrally-resolved dynamic investigation, laser emission was first introduced into a monochromator to extract emission within a wavelength range of FWHM $8 \mathrm{~nm}$. Afterwards, it was received by a high-speed photo detector for measuring laser transient behavior. A rate-equation model based on [5], including quantum-dot GS, ES, and the carrier thermal escape effect, was applied for theoretical study.

Fig. 1 shows the electroluminescence spectra under different injection current densities. GS lasing is observed at 1.3 $\mu \mathrm{m}$, while ES lasing is around $1.22 \mu \mathrm{m}$. At the lowest excitation current, excited states do not contribute much to the whole emission while ground states have shown lasing already. This is a clear evidence that the two emission peaks originate from ground states and excited states respectively, but not from two QD groups of different sizes.

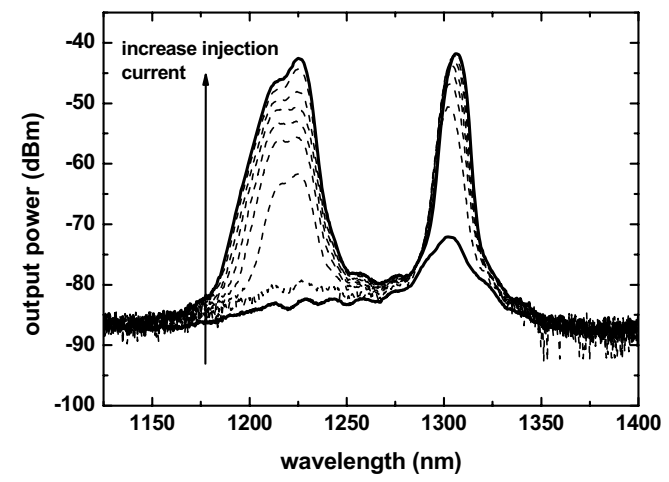

Fig 1. Electroluminescence spectra under various injection current densities. Two emission peaks are identified as QD ground-state emission at $1.3 \mu \mathrm{m}$ and QD excited-state emission around $1.22 \mu \mathrm{m}$.

Fig 2 shows individual large-signal modulation measurement results from GS around $1.3 \mu \mathrm{m}$ and ES around 1.22 $\mu \mathrm{m}$ respectively. The onset of ES lasing prior to GS lasing is experimentally observed for the first time. This interesting 
feature can be further analyzed with the help of numerical simulation. Fig. 3 shows our theoretical prediction of two-state-lasing dynamics in QD lasers. Excited states can achieve lasing earlier than GS lasing although their emission intensity is lower in the steady state. This phenomenon is consistent with measurement results shown in Fig. 2 and can be explained in the following. When cavity length is shortened, optical loss will be increased approaching GS saturation gain. In order to achieve lasing, more carriers have to be injected into QD ground states and therefore, the relaxation time for carriers from ES into GS will be increased due to Pauli exclusion principle. In rate-equation modeling, this relaxation time is assumed to be inversely proportional to unfilled number of QD ground states [2]. As a result, in steady state, carrier density in ES can be further increased with increasing injection current density even above GS threshold, as shown in Fig. 1. This static two-state-lasing phenomenon has been reported in the literature [2,3,4]. In static case, if there is two-state lasing, ground-state lasing will be always first observed and then, with gradually increased injection current density, ES lasing will be achieved afterwards. However, in transient measurement with an electrical pulse excitation, carrier density in ES can build up very fast when GS occupation probability is relatively high and carrier relaxation time from ES into GS becomes longer and longer. Depending on the optical loss level compared to GS saturation gain, ES lasing can occur prior to GS lasing, as in the cases of Fig. 2 and Fig. 3. Therefore, even when ES emission is small or negligible in steady state, it can be significant for a short period of time before the onset of GS lasing.

In summary, spectrally-resolved dynamics of two-state lasing in quantum-dot lasers is experimentally demonstrated for the first time in this study. The onset of excited-state lasing prior to ground-state lasing is consistent with our theoretical simulation. Under certain conditions, especially when the optical loss level is close to GS saturation gain, ES emission will play a more important role in QD laser transient response than in static measurement.

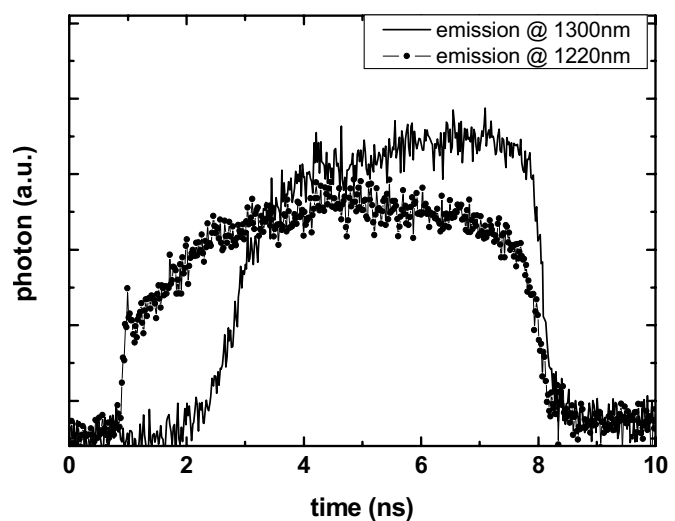

Fig. 2 Measured photon transients under six times threshold current density. Solid curve and circle-line curve represent emission from GS at $1300 \mathrm{~nm}$ and ES at $1220 \mathrm{~nm}$ respectively (FWHM $8 \mathrm{~nm}$ ).

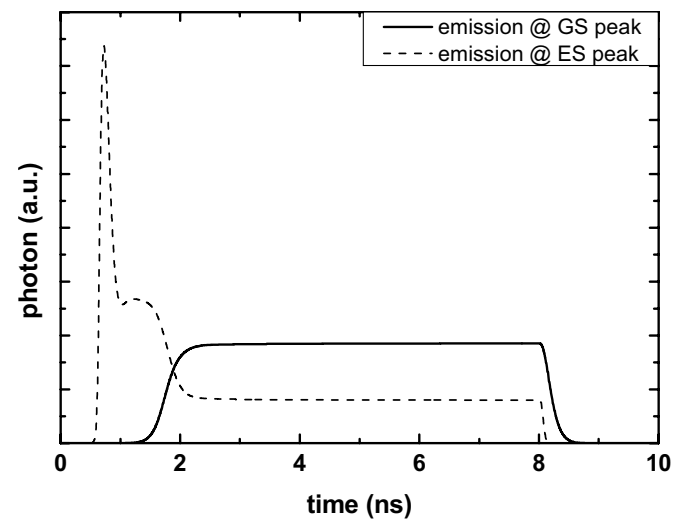

Fig. 3 Simulated photon transients using a rate-equation model.

Acknowledgments: This work was partially supported by the National Science Council, Republic of China, under the Grant No. NSC93-2215-E-002-025. Part of this work has been done with the computation capacity supported by National Center for High-performance Computing, Republic of China.

\section{Reference:}

[1] D. Bimberg, M. Grundmann and N. N. Ledentsov, Quantum Dot Heterostructures. John Wiley and Sons, 1999.

[2] M. Sugawara, N. Hatori, E. Ebe, M. Ishida, Y. Arakawa, T. Akiyama, K. Otsubo and Y. Nakata, "Modeling room-temperature lasing spectra of $1.3 \mu \mathrm{m}$ self-assembled InAs/GaAs quantum-dot lasers: Homogeneous broadening of optical gain under current injection”, J. Appl. Phys. 97, 043523 (2005)

[3] M. V. Maximov, L. V. Asryan, Yu. M. Shernyakov, A. F. Tsatsul'nikov, I. N. Kaiander, V. V. Nikolaev, A. R. Kovsh, S. S. Mikhrin, V. M. Ustinov, A. E. Zhukov, Zh. I. Alferov, N. N. Ledenstov and D. Bimberg, "Gain and Threshold Characteristics of Long Wavelength Lasers Based on InAs/GaAs Quantum Dots Fromed by Activated Alloy Phase Separation", IEEE J. Quantum. Electron. 37, 676 (2001)

[4] A. Markus, J. X. Chen, C. Paranthoen and A.Fiore, "Simultaneous two-state lasing in quantum-dot lasers", Appl. Phys. Lett. 82, $1818(2003)$

[5] M. Sugawara, T. Akiyama, N. Hatori, Y. Nakata, H. Ebe and H. Ishikawa, "Quantum-dot semiconductor optical amplifiers for high-bit-rate signal processing up to $160 \mathrm{~Gb} \mathrm{~s}^{-1}$ and a new scheme of 3R regenerators”, Meas. Sci. Technol. 13, 1683 (2002) 